\title{
Ultrasound assessment of the myofascial trigger point, where is the local twitch response located?
}

\author{
Minaya Muñoz F. ${ }^{1}$ Valera Garrido F. ${ }^{1}$ Pereira Barbosa M. ${ }^{2}$ Espinola Marcos A. ${ }^{3}$ \\ ${ }^{1}$ MVClinic, Madrid, Spain \\ ${ }^{2}$ Clínica FisioOn, Fisioterapia Saúde Integral, Portugal \\ ${ }^{3}$ Centro de Fisioterapia Espinola, Valencia, Spain
}

Rev Fisioter Invasiva 2019;2:119-120.

\begin{abstract}
\section{Keywords}

- ultrasound guided dry needling

- myofascial trigger point

- ultrasound

Background A myofascial trigger point (MTrP) is defined as a nodule or hyperirritable spot of focal pain in response to pressure which is found on a palpable taut band of skeletal muscle. For the physical therapy diagnosis, assessment tools such as musculoskeletal ultrasound have shown that it is possible to visualize the MTrP in gray scale (B mode) and in elastography; however, no previous studies have evaluated the correlation between the appearance of a local twitch response and the presence of a myoaponeurotic septum. The aim of the present study was to evaluate the correlation between the local twitch response and the presence of a myoaponeurotic septum at the time of the response.

Material and Methods A prospective observational study was performed at the Sannus Clinic center (Madrid) including patients with the following inclusion criteria: subjects over the age of 18 , with the presence of active MTrPS according to the criteria established by Travell \& Simons. The ultrasound-guided dry needling technique was performed (US-guided DN) on the dysfunctional muscles, according to the methods described by Valera \& Minaya, as follows: 1) manual identification and localization of the myofascial trigger point; 2) Ultrasound assessment of the area corresponding to the identified MTrP. The palpatory hand was substituted in this case by the ultrasound probe which applies pressure upon this band, for its study; 3 ) Establishment of safety measures during the procedure; 4 ) The extremes of the probe were marked using a dermographic pen; 5) Removal of the probe and asepsis of the skin of the area prior to needling; 6) Pre-intervention measures: hand-washing, use of non-sterile gloves and a probe cover, followed by preparation of the sterile needle and gauze; 7) The probe was placed on the area of study, with the marks established on the skin; 8) Ultrasoundguided intervention; 9) Needle removal and compression during 30 seconds; 9) Reassessment. Once the local twitch response was visualized, the position of the needle tip was marked on the ultrasound image.

Results The sample was comprised of 40 patients ( 23 men; 17 women) who received the US-guided DN procedure in the following muscles: upper trapezius $(n=32)$, extensor digitorum $(n=25)$, infraspinatus $(n=14)$, medial gastrocnemius $(n=8)$, gluteus medius $(n=12)$. In $87.9 \%$ of muscles $(n=80)$ a local twitch response was obtained when the needle tip was close to the myoaponeurotic septum.
\end{abstract}

Copyright @ 2019 by Thieme Revinter Publicações Ltda, Rio de Janeiro, Brazil 
Discussion From our point of view, the myoaponeurotic septum of a muscle with an active MTrP could represent a place for load transmission, which would justify the activation of the muscle tissue, and the genesis of the MTrP. Considering the diagnostic criteria of the MTrP, the local twitch response is one of the most determinant elements, therefore, its location in the proximity of this anatomical area could be used as a reference.

Conclusions The local twitch response is often obtained in the proximity of a myoaponeurotic septum of the dysfunctional muscle. 